\title{
Winsor \& Newton original handbooks: a surface-enhanced Raman scattering (SERS) and Raman spectral database of dyes from modern watercolor pigments
}

Federica Pozzi ${ }^{1,2,3^{*}}$, John R Lombardi ${ }^{2}$ and Marco Leona ${ }^{1}$

\begin{abstract}
Background: Winsor \& Newton Ltd. has been one of the main fine art products providers since its establishment in 1832, being responsible for the manufacture of a wide assortment of materials ranging from oils and pigments to brushes and papers. All the items produced over the years have been indexed in a comprehensive historical archive. Original Winsor \& Newton handbooks are a powerful resource which can offer insight into the world of artists' materials, and knowledge of artists' choices through the identification of substances employed to obtain particular colors. Scientific analyses of various kinds have been carried out on Winsor \& Newton art materials over the years; however, a detailed study of the organic dyes contained in the watercolors manufactured by the company has never been performed thus far to our knowledge.

Results: In the present study, we examined a number of color washes on drawing paper from two historical Winsor \& Newton catalogues dating to the 19th and 20th century. An appropriate database was thus built, including surface-enhanced Raman scattering (SERS) and Raman spectra of organic colorants from a wide variety of shades. While the selection of colors offered by the company in the 19th century mostly included lakes prepared from plant and insect dyes, i.e. madder and cochineal, some tints based on synthetic dyes were also found in the 20th century handbook.
\end{abstract}

Conclusions: The present article sheds new light on the chemical composition of a number of original Winsor \& Newton color washes in terms of organic colorants contained in each shade. A special attention was dedicated to the analysis of those colors for which the formulation was ambiguous or not specified by the manufacturers, such as dragons' blood and most of the alizarin-based pigments. In addition, we were able to correct erroneous indications provided by Winsor \& Newton on the composition of some tints, as in the case of violet carmine, and study how the formulation of certain pigments has been modified over the centuries.

Keywords: Winsor \& Newton, Historical catalogues, Watercolor pigments, Organic colorants, Surface-enhanced Raman spectroscopy, Raman spectroscopy

\footnotetext{
* Correspondence: fpozzi@artic.edu

'Department of Scientific Research, The Metropolitan Museum of Art

New York, NY, USA

²Department of Chemistry, The City College of New York, CUNY, New York,

NY, USA

Full list of author information is available at the end of the article
} 


\section{Introduction}

Founded in 1832, Winsor \& Newton Ltd. has always been one of the main art materials suppliers in the world, manufacturing a wide assortment of fine art products such as oils, alkyds, watercolors, acrylics, pastels, brushes, canvases and papers. Since the company was established, an extensive archive has been created, including bound records of processes and shopfloor accounts, as well as handwritten books of recipes and notes for making artists' pigments, oil colors, watercolors and a great variety of other art materials. The 19th century Winsor \& Newton archive, containing 87 manuscripts for an overall amount of 17,000 pages, is considered to be the most comprehensive historical collection of this kind dating to the 1800 s [1].

Winsor \& Newton catalogues, with their collections of swatches showing the results obtainable with the firm's colors, are an important historical and scientific resource, which affords scholars a precious insight into the world of artists' materials. Information provided by chemical examination of colors from historical handbooks can be of utmost importance for interpreting analytical data from actual paintings in technical studies or authentication efforts. Understanding whether an artist decided to overlap a given set of colors to obtain a particular shade or if such mixture had been already created by the paint supplier is essential to expand our comprehension of the artist's choices and to shed new light on the techniques employed. Furthermore, chemical analysis applied to the study of original art products may also contribute to set up suitable conservation and restoration approaches, as paint defects as well as the deterioration degree of pigments in works of art can be deeply characterized and properly treated by examining actual recipes for a certain color.

Among several analytical techniques currently employed in the study of art materials, surface-enhanced Raman spectroscopy (SERS) has proven to be one of the most suitable for characterizing organic dyes [2], which are typically very difficult to identify by normal Raman spectroscopy because of their high fluorescence emission. In SERS, dyes are adsorbed onto nanosized metal substrates such as silver colloids, and this provides greatly enhanced Raman signals and fluorescence quencing, thus allowing for the ultrasensitive detection of trace amounts of analytes [3].

A few studies recently published in the literature describe the analysis of Winsor \& Newton acrylic, alkyd paints and watercolor pigment cakes by gas chromatography mass spectrometry (GC-MS), attenuated total reflection Fourier transform infrared spectroscopy (ATR-FTIR), matrix-assisted laser desorption/ionization - mass spectrometry (MALDI-MS), electrospray ionization - mass spectrometry (ESI-MS), X-ray diffraction (XRD), and normal Raman spectroscopy [4-7]. In recent times, a SERS characterization of four color washes from a Winsor \& Newton catalogue has been also performed in order to provide reference spectra for the identification of specific colorants in a watercolor by the American print-maker and painter Homer [8]. However, to the best of our knowledge, a comprehensive Raman and SERS study of dyes from historical Winsor \& Newton handbooks has to date never been carried out.

In the present work, we examined several color washes from two original Winsor \& Newton catalogues from the 19th and 20th century collections $[9,10]$, aiming to identify the organic colorants possibly contained in each shade. Figure 1 displays a selection of swatches from one of the historical handbooks under investigation, where pigments have been arranged by the manufacturers in order to bring out their color and emphasize the chromatic effect obtainable by their juxtaposition. A spectral database of original art materials was thus acquired, including SERS and Raman spectra of several pink, red, violet, brown and gray shades. Based on the observed

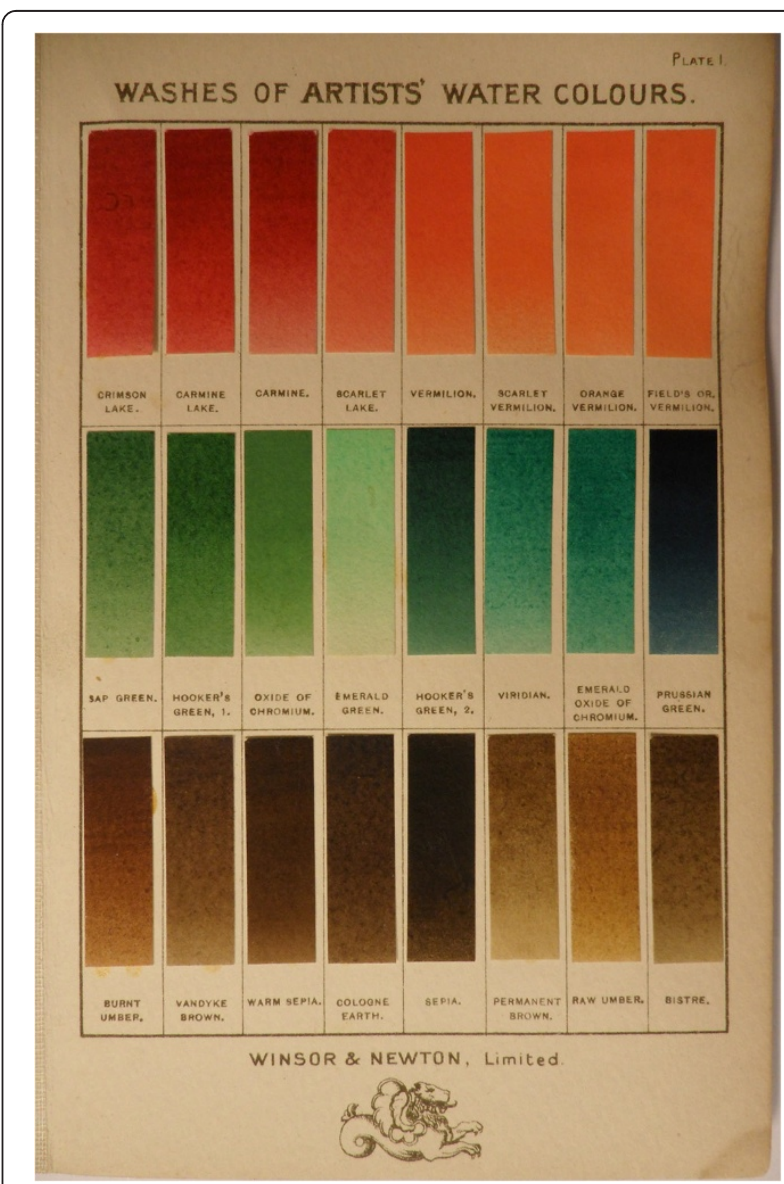

Figure 1 Winsor \& Newton 20th century catalogue. Selection of color washes from the 20th century Winsor \& Newton handbook of watercolor pigments under investigation. 
spectral patterns, Winsor \& Newton pigments were divided into four different categories, and their chemical composition was discussed in connection with the preparation procedures declared by the manufacturers. The results reported in this article may represent valuable reference data to be used for dating, as well as in authentication and identification studies.

\section{Experimental \\ Materials}

Silver nitrate, sodium citrate, sulfuric acid, glucose and hydrofluoric acid were purchased from Fisher Scientific; madder lake (lake of alizarin/purpurin), carmine naccarat (alumina lake of carminic acid) and indigo from Kremer Pigments; alizarin, carminic acid, ethanol and potassium nitrate were obtained from Sigma-Aldrich. All the aqueous solutions used for the silver nanoparticle synthesis were prepared using $18 \mathrm{M} \Omega$ ultrapure water (Millipore Simplicity 185 water purification system).

\section{SERS methods: HF hydrolysis, Ag colloid synthesis and sample preparation}

Since most lake pigments are manufactured by precipitating a dye with a metal salt, SERS analysis of Winsor \& Newton colors was carried out following hydrolysis with HF. This step is designed to hydrolyze the dye-metal complexes and maximize dye adsorption onto the colloid surface, in order to obtain the greatest signal enhancement. For this purpose, microscopic samples of Winsor \& Newton lakes on paper, typically smaller than $0.3 \mathrm{x}$ $0.3 \mathrm{~mm}$ in size, were taken using a tungsten needle. HF hydrolysis was then performed by exposing the samples to $\mathrm{HF}$ vapor in a closed microchamber for 5 minutes, according to an optimized procedure previously reported in the literature [11] and explored in detail elsewhere [12].

Silver nanoparticles prepared by microwave-supported glucose reduction of silver sulfate in the presence of sodium citrate as a capping agent were used as a SERS substrate. The synthesis was carried out according to a previously published procedure [13].

Reference solutions of alizarin and carminic acid were prepared in ethanol at a concentration of $10^{-4} \mathrm{M}$, and aliquots of $6 \mathrm{M} \mathrm{HNO}_{3}$ were employed to adjust the $\mathrm{pH}$. For SERS analyses, $0.2 \mu \mathrm{L}$ of the dye solution were added to $0.8 \mu \mathrm{L}$ of the $\mathrm{Ag}$ colloid, followed by the addition of $0.1 \mu \mathrm{L}$ of a $0.5 \mathrm{M} \mathrm{KNO}_{3}$ aqueous solution to induce aggregation of the nanoparticles. On the other hand, reference madder and carmine lakes as well as all the pigments from the two Winsor \& Newton catalogues under investigation were analyzed upon deposition of $0.8 \mu \mathrm{L}$ of the $\mathrm{Ag}$ colloid and $0.1 \mu \mathrm{L}$ of a $0.5 \mathrm{M} \mathrm{KNO}_{3}$ aqueous solution after HF hydrolysis.

\section{Instrumentation}

Normal Raman experiments were performed using a Bruker Senterra Raman spectrometer equipped with an Olympus 100x long working distance microscope objective and a charge-coupled device (CCD) detector. A Spectra Physics Cyan solid state laser and a continuous wave diode laser, emitting light at $488 \mathrm{~nm}$ and $785 \mathrm{~nm}$ respectively, were used as the excitation sources, and two holographic gratings provided a spectral resolution of $3-5 \mathrm{~cm}^{-1}$ (1800 rulings/mm for the $488 \mathrm{~nm}$ laser, $1200 \mathrm{rulings} / \mathrm{mm}$ for the $785 \mathrm{~nm}$ laser). Output laser powers of 0.25 or $2.5 \mathrm{~mW}$ for $488 \mathrm{~nm}$ excitation and 10 or $25 \mathrm{~mW}$ for $785 \mathrm{~nm}$ excitation were employed for the analysis, according to the Raman response of the different pigments.

SERS spectra were recorded with the same Bruker Senterra Raman instrument, using a 20x long working distance microscope objective and excitation at $488 \mathrm{~nm}$, with a single integration of $30 \mathrm{~s}$. SERS analyses were executed by focusing onto the microaggregates which were formed inside the dye-colloid droplet a few seconds after the deposition of the $\mathrm{Ag}$ nanoparticles and $\mathrm{KNO}_{3}$.

\section{Results and discussion}

Detailed results obtained from the SERS and Raman analyses of pink, red, violet, brown and gray shades from the two Winsor \& Newton handbooks under investigation are presented in this section. Spectra collected from a number of colors (solid lines) were compared with those of reference pure dyes and lakes (dashed lines) for identification purposes, and the pigments examined could be thus divided into different classes according to the outcomes of spectroscopic analysis. It is worth pointing out that the chemical composition is declared by Winsor \& Newton only for some of the color washes included in the catalogues; the trade names given to each shade by the manufacturers are typically indicative of the color rather than the chemical constituents.

Spectra of lakes belonging to the same class were found to be often characterized by slight shifts in wavenumber and changes in relative intensities, which can be attributed to complexation of the dyes with different metal ions in the lake manufacture [8].

\section{Cochineal-based pigments}

A first group of pigments is composed of crimson lake, carmine lake, carmine, burnt carmine, purple lake and Indian purple, all ranging from dark red to purple shades. According to Winsor \& Newton, all these colors were prepared by precipitating the extract of cochineal, an insect red dye indigenous to Mexico and South America, with different amounts of inorganic substrates. Analytical results confirmed for these lake pigments a cochineal-based composition in both the 19th century and 20th century editions (as an example, see spectra of crimson lake in 
Figures 2 and 3). SERS spectra upon HF hydrolysis display the typical spectral pattern of the main component of cochineal, i.e. carminic acid, which is detected after acid treatment as a free colorant. In particular, signals around $1635,1579,1448,1324,1222,1069$ and $449 \mathrm{~cm}^{-1}$ show a remarkable correspondence with those of a reference carminic acid solution (Figure 2). Raman spectra acquired from these lakes at $488 \mathrm{~nm}$ excitation exhibit common features at 1641, 1479, 1318 and $1109 \mathrm{~cm}^{-1}$, which match the main Raman bands of carmine naccarat from Kremer Pigments (Figure 3).

In the catalogue belonging to the 19th century collection, four additional color washes were found to be based on carminic acid, i.e. scarlet lake, neutral tint, Payne's grey and violet carmine. Scarlet lake is described by Winsor \& Newton as an intimate combination of crimson lake with pale vermillion. Accordingly, the typical Raman signals of carmine were observed at $488 \mathrm{~nm}$ (Figure 3), while vermillion could be identified by its distinctive bands at 343, 283 and $253 \mathrm{~cm}^{-1}$ using the $785 \mathrm{~nm}$ laser line. The detection of carmine was also supported by the SERS spectrum, in which the characterizing lines of carminic acid arose following HF hydrolysis (Figure 2). For what concerns neutral tint, its chemical composition has not been described in the catalogues; however, this pigment is reported in the Colour Index to be a mixture of Indian ink - which is composed of a variety of fine soot and the inorganic pigment Prussian blue, together with a very small amount of madder lake. Also, according to

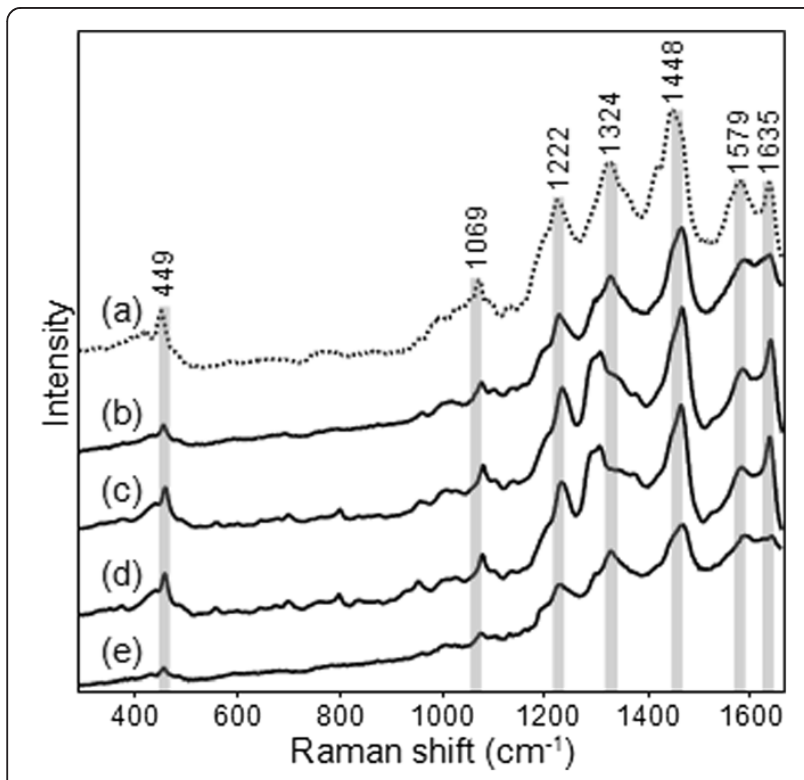

Figure 2 SERS analysis of cochineal-based pigments. (a) SERS spectrum of reference carminic acid compared to examples of SERS spectra obtained from Winsor \& Newton cochineal-based pigments $\left(\lambda_{\text {exc }}=488 \mathrm{~nm}\right)$ : (b) crimson lake 20th century edition, (c) crimson lake 19th century edition, (d) scarlet lake 19th century edition, and (e) dragons' blood 20th century edition.

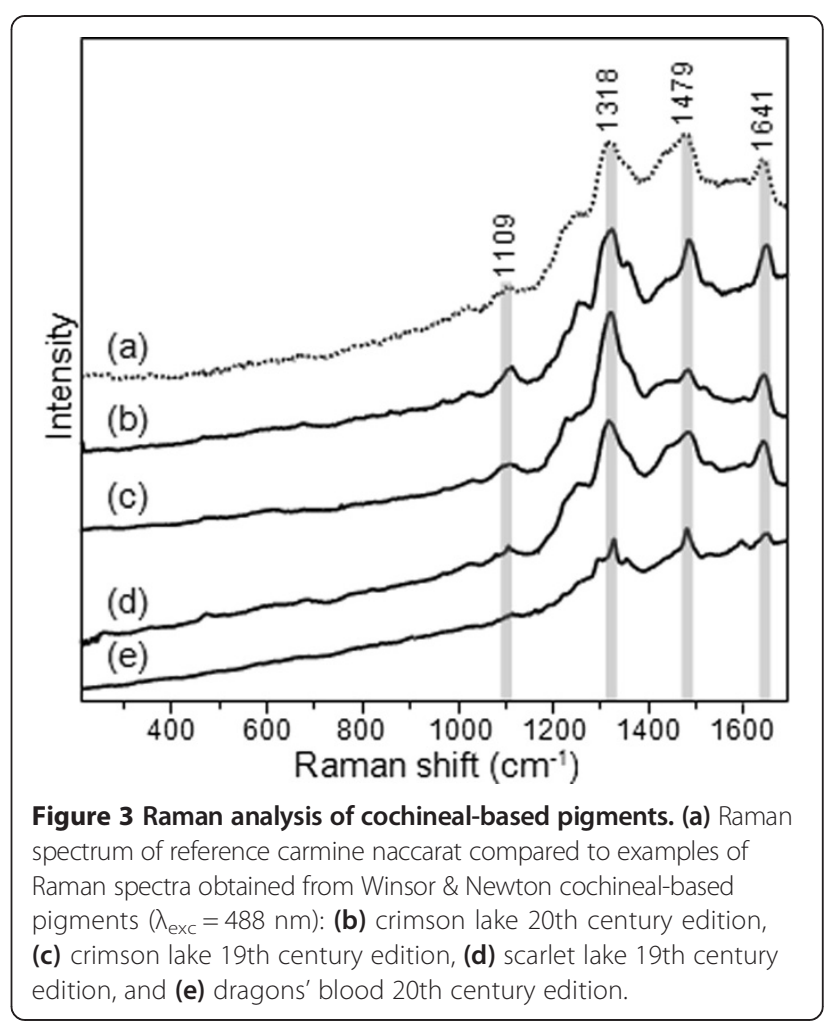

Winsor \& Newton, Payne's grey is supposed to have a composition similar to that of neutral tint and, even if more lilac in hue, to resemble its properties. However, for both these pigments a good spectrum of carminic acid was obtained by SERS, while Raman spectroscopy detected carmine at $488 \mathrm{~nm}$ (see Figures 4 and 5 for 19 th century neutral tint). Surprisingly, the characteristic signals of indigo were also identified by Raman at $488 \mathrm{~nm}$ (for Payne's grey only) and $785 \mathrm{~nm}$ (for both samples) (Figure 5), while no traces of Prussian blue were detected. Finally, violet carmine is described in the catalogues as a lake obtained from the roots of Anchusa tinctoria, a tinctorial plant from which alkanet, a red colorant with naphthoquinonic chromophores, is extracted. However, SERS allowed us to detect carminic acid in this color wash as well. Most importantly, Raman and SERS analyses demonstrated that in all these four pigments discussed above - scarlet lake, neutral tint, Payne's grey and violet carmine - carminic acid was replaced with synthetic alizarin in the 20th century Winsor \& Newton catalogue. A detailed comparison of SERS and Raman spectra of neutral tint from the 19th and 20th century editions is reported in Figures 4 and 5.

Particularly interesting is the case of the dragons' blood color wash, only present in the 20th century edition studied. The name "dragons' blood" has been used since antiquity with reference to a red natural resin native of East Indies which was traditionally extracted from 


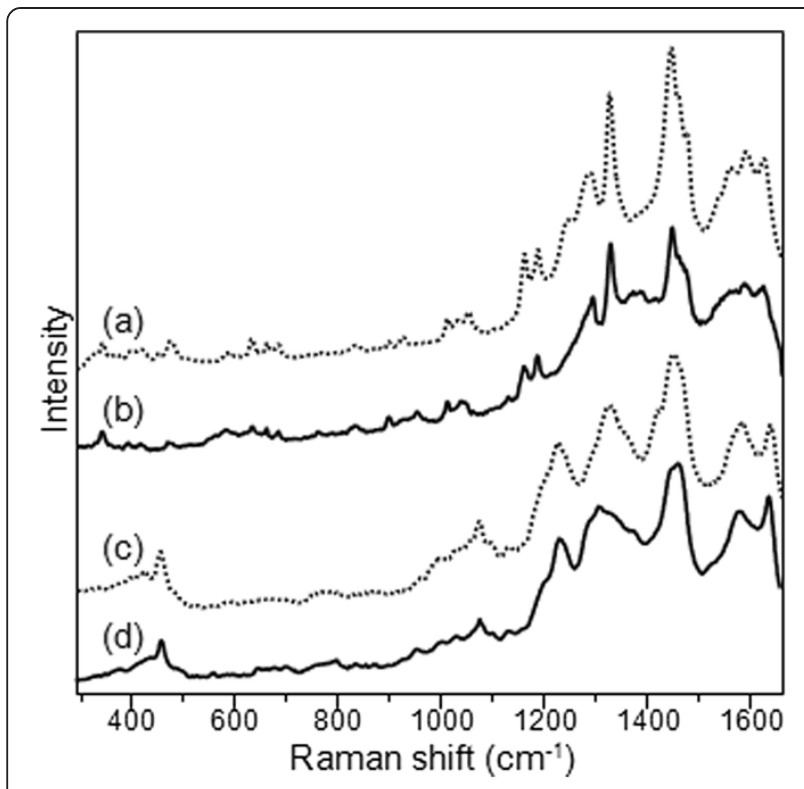

Figure 4 SERS analysis of neutral tint. (a) SERS spectrum of reference alizarin compared to (b) that of Winsor \& Newton neutral tint 20th century edition, and (c) SERS spectrum of reference carminic acid compared to (d) that of Winsor \& Newton neutral tint 19th century edition $\left(\lambda_{\text {exc }}=488 \mathrm{~nm}\right)$. The most distinctive SERS peaks of alizarin (spectra $\mathbf{a}, \mathbf{b}$ ) and carminic acid (spectra $\mathbf{c}, \mathbf{d}$ ) are indicated in Figures 7 and 2, respectively.

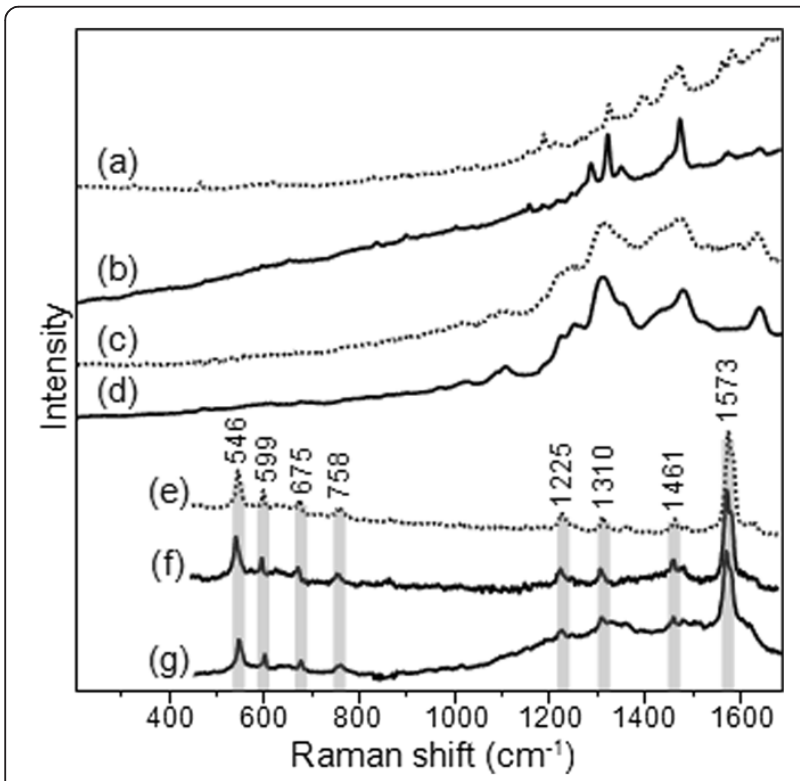

Figure 5 Raman analysis of neutral tint. (a) Raman spectrum of reference alizarin compared to (b) that of Winsor \& Newton neutral tint 20th century edition, and (c) Raman spectrum of reference carmine naccarat compared to (d) that of Winsor \& Newton neutral tint 19th century edition $\left(\lambda_{\text {exc }}=488 \mathrm{~nm}\right.$ ). (e) Raman spectrum of reference indigo compared to those of Winsor \& Newton neutral tint (f) 20th century edition and $\mathbf{( g )}$ 19th century edition $\left(\lambda_{\text {exc }}=785\right.$ $\mathrm{nm}$ ). The most distinctive Raman peaks of alizarin (spectra $\mathbf{a}, \mathbf{b}$ ) and carmine (spectra $\mathbf{c}, \mathbf{d}$ ) are indicated in Figures 8 and 3, respectively. plants belonging to the Dracaena and Daemonorops genera. However, as also pointed out by Burgio [4], such dye was replaced in the 19th century by a more lightfast one due to its lack of permanence upon exposure to light. Accordingly, Winsor \& Newton dragons' blood is described as an imitative colorant which is a semipermanent substitute of the original homonymous pigment. However, more detailed information concerning its chemical composition was not provided. Spectroscopic analyses allowed us to number Winsor \& Newton's dragons' blood among cochineal-based colors, as both its SERS and Raman spectra are in agreement with the results obtained for the other shades of this category (Figures 2 and 3). In particular, the Raman spectrum of this shade shows a remarkable resemblance with that acquired by Burgio from a dragons' blood pigment cake belonging to a 19th century Winsor \& Newton watercolor box [4], suggesting an analogous composition for these two materials.

\section{Madder lake-based pigments}

A second wide class of colors consists of madder carmine, madder lake, rose madder, pink madder, brown madder, Rubens' madder and purple madder. These pigments are reported by Winsor \& Newton to have been manufactured by precipitating the extract of madder roots mostly in combination with alumina. Alizarin and purpurin are the main component of the madder dye, well-known in antiquity and traditionally extracted from the roots of Rubia tinctorum. Accordingly, SERS spectra upon HF treatment display intense signals at 1575, 1444, 1326, 1272, 1158 and $1015 \mathrm{~cm}^{-1}$, well corresponding to those of reference madder lake purchased from Kremer Pigments and subjected to the same kind of hydrolysis procedure (Figure 6). Such composition in terms of organic dyes has been confirmed for the pigments listed above in both the catalogues studied (as an example, see spectra of rose madder and Rubens' madder in Figure 6). In addition, lakes such as permanent brown, rose madder pk shade, scarlet madder and rose dorè, for which no chemical information was provided either in the Winsor \& Newton catalogues or in the Colour Index, could be also included in the category here discussed based on the outcomes of SERS analysis (Figure 6). All the pigments of the present class were found to be very fluorescent when analyzed by normal Raman spectroscopy. However, in a few cases typical signals of madder lake at 1476 and $1323 \mathrm{~cm}^{-1}$ could be identified in the spectra (data not shown).

As clearly suggested by SERS, madder lake was used for warm sepia as well. This dark brown pigment is described by Winsor \& Newton as a mixture of a secretion of the cuttlefish, Sepia officinalis, with browns of a red hue, the composition of which was not specified by the 


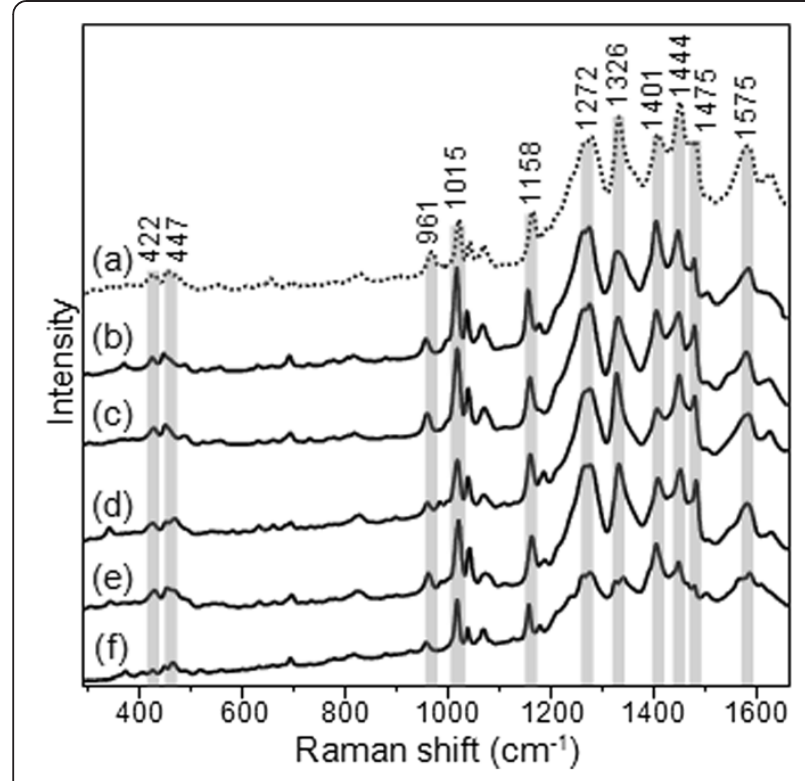

Figure 6 SERS analysis of madder lake-based pigments. (a) SERS spectrum of reference madder lake upon HF treatment compared to examples of SERS spectra obtained from Winsor \& Newton madder lake-based pigments $\left(\lambda_{\text {exc }}=488 \mathrm{~nm}\right.$ ): (b) rose madder 20th century edition, (c) rose madder 19th century edition, (d) Rubens' madder 20th century edition, (e) Rubens' madder 19th century edition, and (f) warm sepia 20th century edition.

manufacturers, but could be here ascertained by means of spectroscopic analyses (Figure 6).

\section{Alizarin-based pigments}

Besides pigments based on insect and plant dyes such as cochineal and madder, high quality SERS and Raman spectra could also be obtained for some color washes based on synthetic colorants from the 20th century Winsor and Newton catalogue. Specifically, rose madder (alizarin), alizarin scarlet, scarlet madder (alizarin), alizarin carmine, alizarin crimson, ruby madder (alizarin), purple madder (alizarin), permanent crimson and madder carmine (alizarin) were found to have been produced from alizarin. SERS spectra upon HF treatment exhibit bands at 1624, 1587, 1558, 1448, 1324, 1287, 1185 and $1158 \mathrm{~cm}^{-1}$ which are in good agreement with those of reference alizarin solution (Figure 7). For all the Winsor \& Newton lakes belonging to the present class, resonance Raman spectra at $488 \mathrm{~nm}$ excitation with common features at 1477, 1350, 1325 and $1288 \mathrm{~cm}^{-1}$ were obtained, matching well the two most intense signals at 1477 and $1325 \mathrm{~cm}^{-1}$ arising in the Raman spectra of reference alizarin (Figure 8). The fact that this dye was only found in the 20th century edition suggests that probably it was not obtained from the madder plant, but it is of synthetic origin.

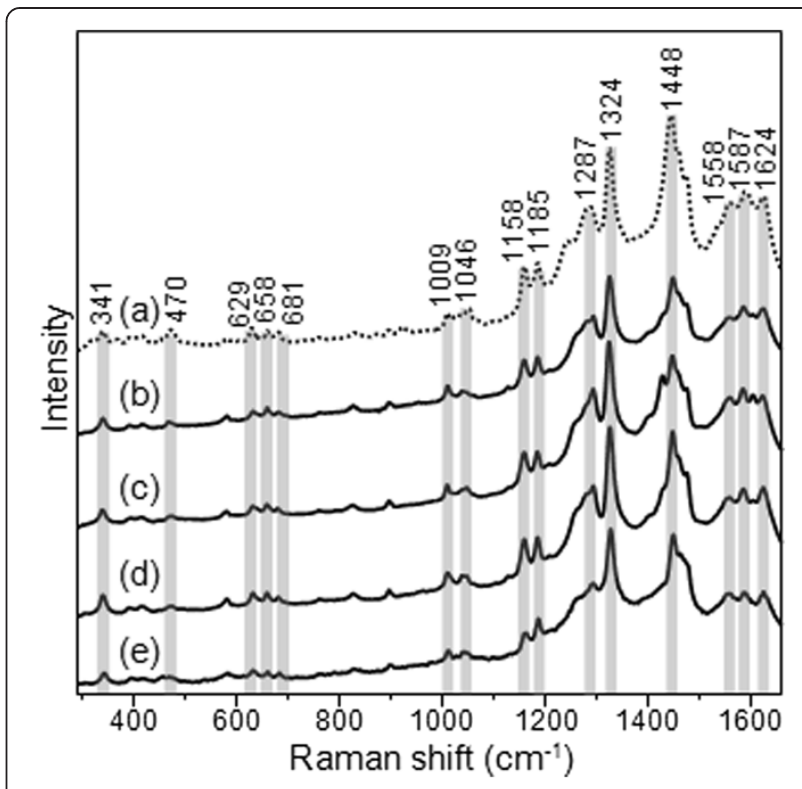

Figure 7 SERS analysis of alizarin-based pigments. (a) SERS spectrum of reference alizarin compared to examples of SERS spectra obtained from Winsor \& Newton alizarin-based pigments from the 20th century edition $\left(\lambda_{\text {exc }}=488 \mathrm{~nm}\right.$ ): (b) ruby madder (alizarin), (c) scarlet lake, (d) permanent crimson, and (e) violet carmine.

In addition, as anticipated in a previous paragraph, scarlet lake, neutral tint, Payne's grey and violet carmine were found to have a different chemical composition in the two Winsor \& Newton catalogues studied. Indeed, while in the 19th century edition these color

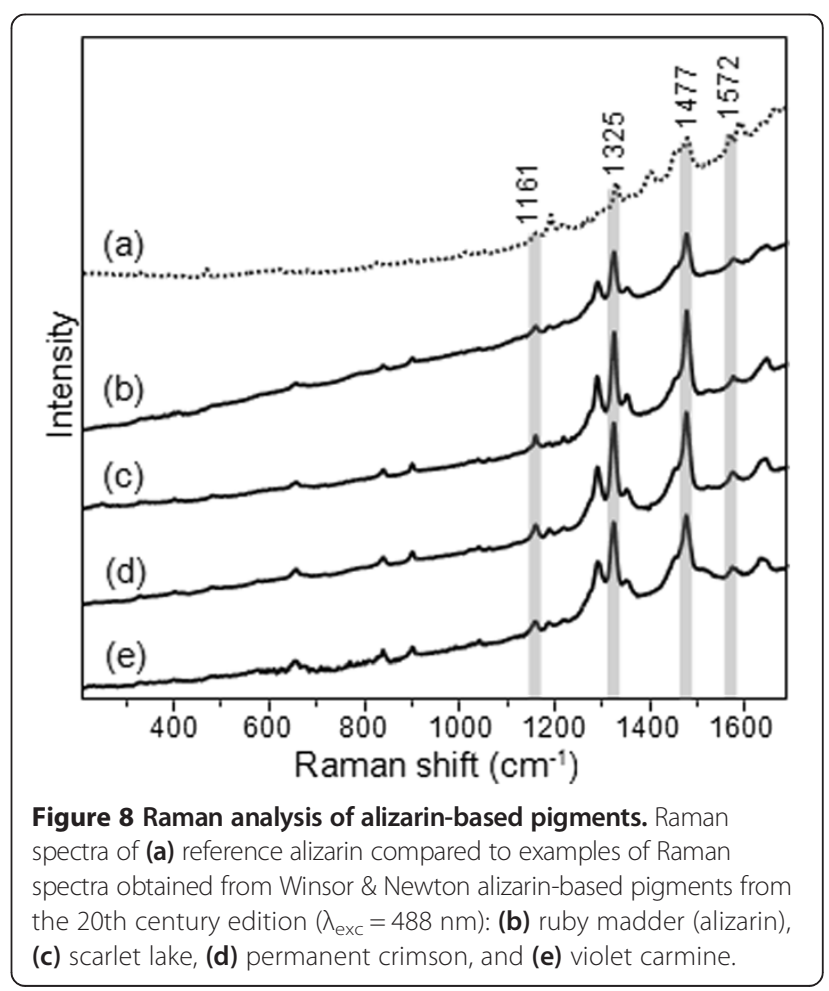


washes were mainly based on cochineal, in the 20th century edition they were found to be made of alizarin (Figures 7 and 8).

\section{Various synthetic pigments}

A few violet and red colors based on synthetic dyes different from alizarin were also found in the 20th century Winsor \& Newton handbook examined in this article.

Mauve, described in the catalogue as a lake prepared from aniline, displayed very intense SERS signals at 1621 , $1590,1371,1176,914$ and $806 \mathrm{~cm}^{-1}$, well corresponding to the spectral pattern obtained for the reference synthetic dyes crystal violet, $\mathrm{N}$-hexamethylpararosaniline, or methyl violet (Figure 9). Because the two dyes have analogous molecular structures, which only differ in the number of methyl groups, it is not possible to differentiate between them by SERS, as their spectra look identical [14].

The use of synthetic dyes was also highlighted in the case of spectrum red by Raman spectroscopy, using 488 and $785 \mathrm{~nm}$ laser lines for excitation. In both cases the observed spectral patterns essentially correspond to the Raman spectrum of the beta-naphthol pigment 1-(4-methyl-2-nitrophenylazo)-2-naphthol, commercially named Pigment Red 3 or Hansa Scarlet RNC (Figure 10).

\section{Conclusions}

In the present study, we examined pink, red, violet, brown and gray lakes on drawing paper from two historical Winsor \& Newton handbooks of watercolor pigments

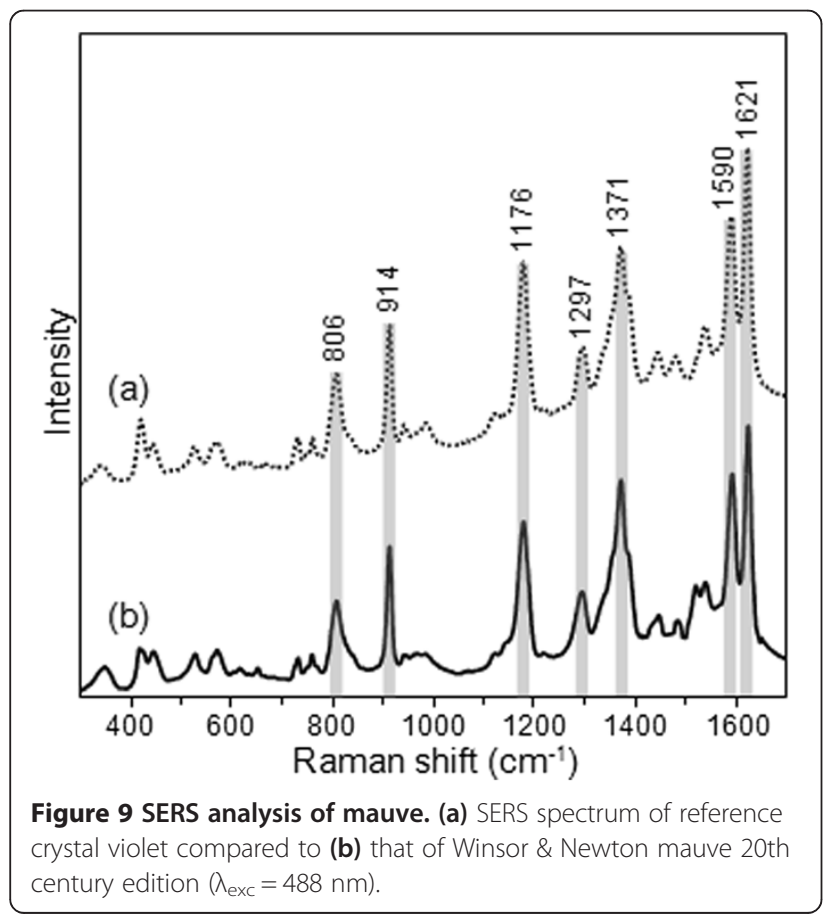

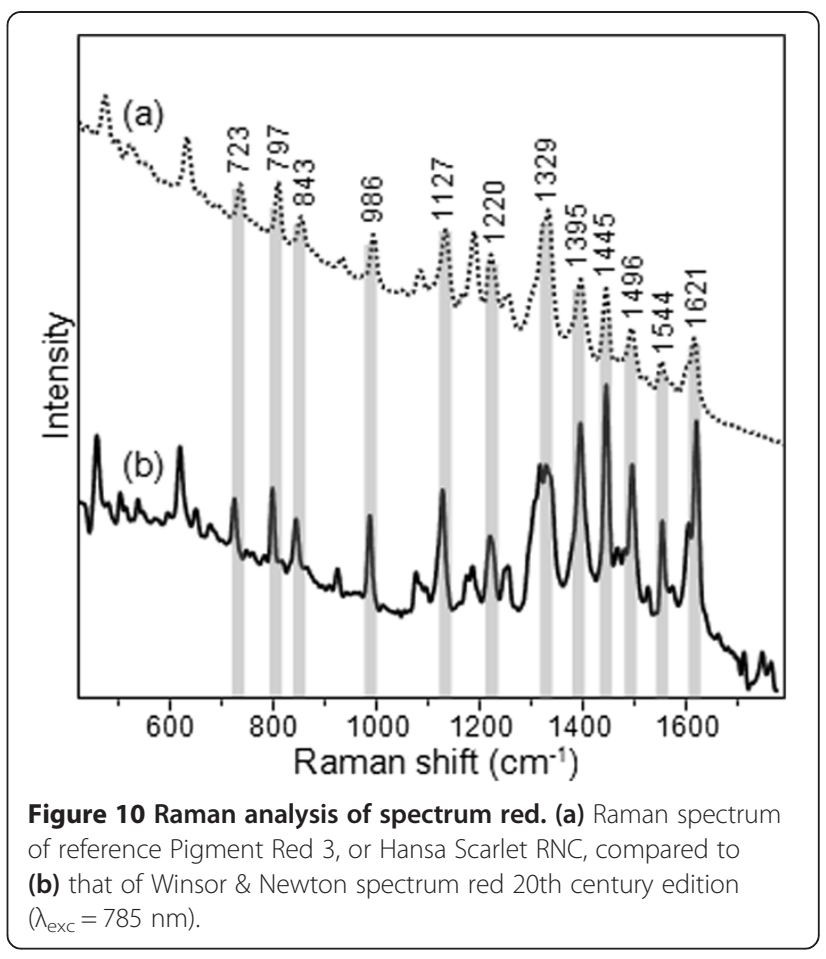

belonging to the 19th and 20th century collections, aiming to identify the organic colorants contained in each of them. An appropriate database was built, including both normal Raman and SERS spectra for a wide number of shades. The so obtained spectral patterns were examined, compared to reference spectra of pure colorants and lake pigments, divided into different groups, and thus discussed.

Spectroscopic analysis of Winsor \& Newton color washes allowed us to deepen our knowledge about the variety of pigments in use during the modern age, and the results obtained provide valuable reference data for dating, as well as in authentication and identification studies. While lakes prepared from plant and insect dyes, mostly madder and cochineal, are predominant among the colors offered by Winsor \& Newton in the 19 th century, some tints based on synthetic dyes such as alizarin were also found in the 20th century edition, often accompanied by commentary on their poor lightfastness. The present Raman and SERS study allowed us to formulate some hypotheses concerning the main constituents of those shades for which a description is not available in the handbooks, such as dragons' blood and most of the alizarin-based pigments. In addition, we could correct erroneous indications provided by Winsor \& Newton on some tints, as in the case of violet carmine, and study how the formulation of certain pigments has been modified over the time. 


\section{Competing interests}

The authors declare that they have no competing interest.

\section{Authors' contributions}

ML conceived the study. FP performed the analyses, carried out interpretation of the results and drafted the manuscript. Both $\mathrm{ML}$ and JRL participated in the coordination of the study, and helped to draft the manuscript. All authors read and approved the final manuscript.

\section{Acknowledgements}

This project was supported by NSF SCIART Award CHE-1041832. We also acknowledge Award No. 2006-DN-BX-K034, National Institute of Justice, Office of Justice Programs, US Department of Justice. The opinions, findings, and conclusions or recommendations expressed in this publication are those of the authors and do not necessarily reflect those of the Department of Justice. A special thanks to Dr. Jacob Simon (National Portrait Gallery, London, UK) for useful discussion about dating of Winsor \& Newton historical handbooks.

\section{Author details}

'Department of Scientific Research, The Metropolitan Museum of Art, New York, NY, USA. '2Department of Chemistry, The City College of New York, CUNY, New York, NY, USA. ${ }^{3}$ Current address: Department of Conservation Science, Art Institute of Chicago, Chicago, IL, USA.

Received: 23 May 2013 Accepted: 25 July 2013

Published: 29 July 2013

\section{References}

1. Winsor \& Newton - The World's Finest Artists' Materials. wuw.winsornewton.com.

2. Casadio F, Leona M, Lombardi JR, Van Duyne R: Identification of organic colorants in fibers, paints, and glazes by surface enhanced Raman spectroscopy. Accounts of Chemical Research 2010, 43(6):782-791.

3. Aroca R: Surface-Enhanced Vibrational Spectroscopy. Chichester, UK: John Wiley \& Sons; 2006

4. Burgio L, Clark RJH, Martin G, Pantos E, Roberts MA: A multidisciplinary approach to pigment analysis: king's yellow and dragon's blood from the Winsor and Newton pigment box at the Victoria and Albert Museum. NATO Science Series II: Mathematics, Physics and Chemistry 2003, 117:61-72.

5. Hoogland FG, Boon JJ: Analytical mass spectrometry of poly(ethylene glycol) additives in artists acrylic emulsion media, artists paints, and microsamples from acrylic paintings using MALDI-MS and nanospray-ESI-MS. International Journal of Mass Spectrometry 2009, 284:72-80.

6. Pintus $V$, Wei $S$, Schreiner M: UV ageing studies: evaluation of lightfastness declarations of commercial acrylic paints. Anal Bioanal Chem 2011 402(4):1567-1584.

7. Ploeger R, Musso S, Chiantore O: Contact angle measurements to determine the rate of surface oxidation of artists' alkyd paints during accelerated photo-ageing. Prog Org Coat 2009, 65:77-83.

8. Brosseau CL, Casadio F, Van Duyne RP: Revealing the invisible: using surface-enhanced Raman spectroscopy to identify minute remnants of color in Winslow Homer's colorless skies. Journal of Raman Spectroscopy 2011, 42:1305-1310.

9. A descriptive handbook of Modern Water Colours, illustrated with actual washes of the pigments on Whatman's drawing paper, with an introductory essay on the recent water-colour controversy by J. Scott Taylor, B.A., Camb. 3rd edition. London, UK: Winsor \& Newton Limited. 1888 [date from first page of catalogue appendix, "Winsor \& Newton's Catalogue of Colours and Materials for Water Colour Painting"].

10. Modern Water-Colour Pigments, Twenty-Fourth Thousand. London, UK: Winsor \& Newton Limited. Undated.

11. Leona M, Stenger J, Ferloni E: Application of surface-enhanced Raman scattering techniques to the ultrasensitive identification of natural dyes in works of art. Journal of Raman Spectroscopy 2006, 37(10):981-992.

12. Pozzi F, Lombardi JR, Bruni S, Leona M: Sample treatment considerations in the analysis of organic colorants by surface-enhanced Raman scattering. Anal Chem 2012, 84:3751-3757.
13. Leona M: Microanalysis of organic pigments and glazes in polychrome works of art by surface-enhanced resonance Raman scattering. Proc Natl Acad Sci 2009, 106(35):14757-14762.

14. Geiman I, Leona M, Lombardi JR: Application of Raman spectroscopy and surface-enhanced Raman scattering to the analysis of synthetic dyes found in ballpoint pen inks. Journal of Forensic Science 2009, 54(4):947-952.

doi:10.1186/2050-7445-1-23

Cite this article as: Pozzi et al:: Winsor \& Newton original handbooks: a surface-enhanced Raman scattering (SERS) and Raman spectral database of dyes from modern watercolor pigments. Heritage Science 2013 1:23.

\section{Publish with ChemistryCentral and every scientist can read your work free of charge \\ "Open access provides opportunities to our colleagues in other parts of the globe, by allowing anyone to view the content free of charge." W. Jeffery Hurst, The Hershey Company.}

- available free of charge to the entire scientific community

- peer reviewed and published immediately upon acceptance

- cited in PubMed and archived on PubMed Central

- yours - you keep the copyright

Submit your manuscript here:

http://www.chemistrycentral.com/manuscript/<smiles>c1ccccc1</smiles>

ChemistryCentral 